\title{
An industrial 3D vision system for size measurement of iron ore green pellets using morphological image segmentation
}

\author{
Matthew J. Thurley *, Tobias Andersson \\ Luleå University of Technology, Dept. of Computer Science \& Electrical Engineering, Luleå \\ SE-97187, SWEDEN
}

\begin{abstract}
An industrial prototype 3D imaging and analysis system has been developed that measures the pellet sieve size distribution into 9 sieve size classes between $5 \mathrm{~mm}$ and $16+\mathrm{mm}$. The system is installed and operational at a pellet production plant capturing and analysing 3D surface data of piled pellets on the conveyor belt. It provides fast, frequent, non-contact, consistent measurement of the pellet sieve size distribution and opens the door to autonomous closed loop control of the pellet balling disk or drum in the future. Segmentation methods based on mathematical morphology are applied to the 3D surface data to identify individual pellets. Determination of the entirely visible pellets is made using a new two feature classification, the advantage being that this system eliminates the resulting bias due to sizing partially visible (overlapped) particles based on their limited visible profile. Literature review highlights that in the area of size measurement of pellets and rocks, no other researchers make this distinction between entirely and partially visible particles. Sizing is performed based on best-fit-rectangle, classified into size classes based on one quarter of the measured sieving samples, and then compared against the remaining sieve samples.
\end{abstract}

Key words: Particle size, On-line analysis

\section{Introduction}

Iron ore mining companies often further refine the extracted ore to produce iron ore pellets which have a uniformly high grade of iron oxide. Iron ore pellets are particularly useful to steel manufacturers as they provide a consistent and high quality iron input to the blast furnace. Variations in pellet size distributions affect the blast furnace process negatively but this affect can be minimised by operating the furnace with different parameters (Ökvist et al., 2001) if the variation in pellet size distribution can be detected.

\footnotetext{
* Corresponding author.

Email address: matthew.thurleyeltu.se (Matthew J. Thurley).

URL: http://www.csee.ltu.se/ mjt (Matthew J. Thurley).
}

For iron ore pellet producers adherence to the target pellet size range is one of the major measures of quality. Green pellets are produced primarily from crushed iron ore in a rotating pelletising disk or tumbling drum after which they are baked in a furnace to produce hardened black pellets.

Existing measurement of green pellet size is a manual sampling and sieving process which is invasive, infrequent, and overly time consuming preventing rapid response to size variation.

The development of an industrial prototype 3D imaging and analysis system is presented for non-contact measurement of the size of green pellets on the conveyor belt. The advantages are frequent sampling and fast generation of sizing results providing rapid feedback to the manufacturing process. 
An imaging system that captures 3D surface data has been implemented based on a projected laser line and camera triangulation (Wikipedia, 2007a, triangulation, structured light). It has a high speed digital camera capable of 4000 frames per second and a continuous wave diode laser with line generating optics. The angle between the camera line of sight and the laser is approximately 24 degrees. The imaging system is installed and operational at a local pellet plant above a conveyor belt moving at two metres per second and collects 3D surface coordinate data at an irregular density of approximately $0.5 \mathrm{~mm}^{2}$.

We note however, that there are a number of sources of error relevant to techniques that measure only what is visible on the surface of a pile;

- Segregation and grouping error, more generally known as the brazil nut effect (Rosato et al., 1987; Wikipedia, 2007c), describes the tendency of the pile to separate into groups of similarly sized particles. It is caused by vibration or motion (for example as rocks are transported by truck or conveyor) with large particles being moved to the surface. Interestingly, iron ore green pellets appear not to be susceptible to this error. Green pellets are wet and relatively sticky and as they travel along they conveyor belt they remain almost entirely fixed in place, with no observable segregation taking place.

- Capturing error (Chavez et al., 1996), describes the varying probability based on size, that a particle will appear on the surface of the pile, Thurley (2002) has explored capturing error in laboratory rock piles but it remains a source of error in this application. Further research is necessary to understand the effect of capturing error for green pellets.

- Profile error, describes the fact that only one side (a profile) of an entirely visible particle can be seen making if difficult to estimate the particles size. However, best-fit-rectangle has been demonstrated as a suitable feature for size classification based on the visible profile (Wang et al., 1996; Wang, 2006) that correlates to sieve size.

- Overlapped particle error, describes the fact that many particles are only partially visible and a large bias to the smaller size classes results if they are treated as small entirely visible particles and sized using only their visible profile. This error can be overcome in piles of particulate material using visibility classification (Thurley, 2002) and an extended two feature classification strategy is presented here.
Literature review indicates numerous publications relating to sizing of particulate material (typically crushed or blasted rock) using imaging. These are predominantly 2D photographic based systems with some of the more prominent being; Maerz et al. (1996); Schleifer and Tessier (2001); Kemeny et al. (2001); Salinas et al. (2005). There are a few publications relating to 3D size measurement; (Blomquist and Wernerson, 1999; Bouajila et al., 1999, iron ore pellets), (Frydendal and Jones, 1998, sugar beets), and (Kim et al., 2003, river rock). However, with the exception of Frydendal and Jones (1998), none of these systems (2D or 3D) removes the bias resulting from overlapped particle error, with Kim et al. (2003) and Lee et al. (2005) recommending a mechanical vibration system to separate rocks and prevent particle overlap. Frydendal and Jones (1998) used graph theory and average region height to determine the entirely visible sugar beets but this approach is insufficient for application to rock piles (Thurley, 2002, 5.5.12) or pellet piles. For pellet sizing, both Blomquist and Wernerson (1999) and Bouajila et al. (1999) assume that pellets are spherical in order to estimate pellet diameter from their measured data. However, we have previously shown that pellets deviate in shape from a sphere such that spherical fitting produces a poor measure of pellet size (Andersson et al., 2007a).

In the presented work we apply 3D surface data analysis based on the work of Thurley and $\mathrm{Ng}(2005)$ to the segmentation and sizing of the data, two feature classification to isolate non-overlapped pellets, sizing based on best-fit-rectangle (Wang et al., 1996; Wang, 2006) to generate a size measure that correlates with sieving, and Nelder and Mead (1965) minimisation to determine decision boundaries for size classes that correlate to a high resolution sieve analysis encompassing 9 size classes between $5 \mathrm{~mm}$ and $16+\mathrm{mm}$.

\section{Segmentation of 3D Surface Data}

\subsection{Background}

The data collected by this system comprises sparse, irregularly spaced 3D coordinate points describing the surface of the pellet pile on the conveyor belt. Data covering approximately one meter of the belt is collected and then analysed. After analysis is completed the process is repeated. Figure 1 shows an enhanced photograph of the laser line on the moving conveyor pellet pile and figure 3 on page 5 shows a portion of the 3D surface of a pellet pile.

There are some inherent complications when it comes to processing 3D surface data collected by laser trian- 
gulation, as follows;

- There are numerous locations where the laser line is not visible from the point of view of the camera. This results in sparse areas in the 3D surface where there are no data points.

- The data is irregularly spaced in the $(\mathrm{x}, \mathrm{y})$ coordinate plane. That is, it is not defined in a regular image space like pixels in a digital camera image.

As a result of these two features of the data we cannot apply image based mathematical morphology operations without first resampling to a regular grid and interpolating to fill in sparse areas.

We note that mathematical morphology is a powerful image processing technique for analysing geometric structure with particular relevance to this problem. Mathematical morphology has applications in edge detection, enhancement, segmentation, reconstruction, texture analysis, particle analysis and shape analysis to name a few areas. The reader is referred to the book Hands-on Morphological Image Processing Dougherty and Lotufo (2003) for an extremely accessible and applied text on mathematical morphology and its applications.

The presented data is sufficiently regular such that it can easily be resampled to a regular grid without the introduction of any noticeable artifacts, however, interpolating the raw data to fill sparse regions is not desirable as it can introduce erroneous artifacts (Thurley et al., 1999) into the data resulting in bias in the analysis.

We therefore present a segmentation and analysis solution based on the mathematical morphology implementation for sparse, irregularly spaced 3D surface

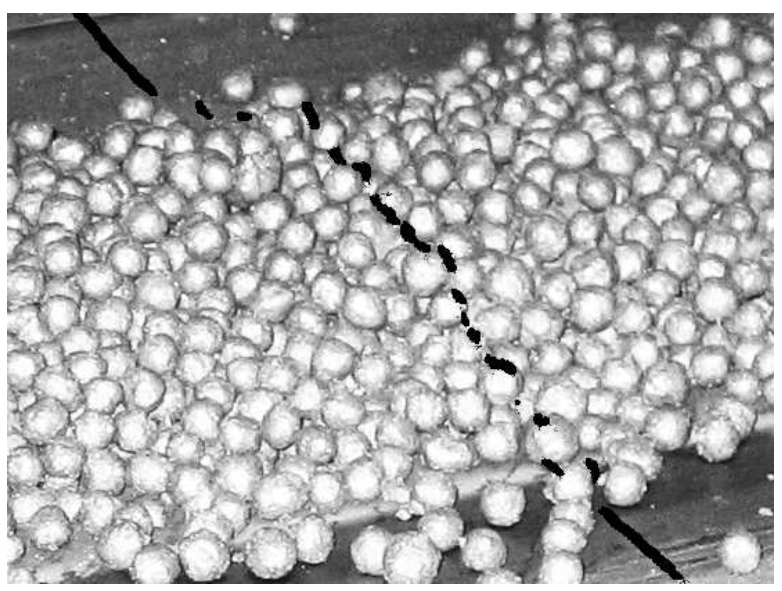

Fig. 1. Laser line across green pellets (enhanced image) data by Thurley and $\mathrm{Ng}$ (2005). As shown by Thurley (2002) the application of this implementation can identify individual rocks on the surface of laboratory rock piles with sufficient accuracy to estimate the pile size distribution. Therefore, we shall use this mathematical morphology implementation as a framework for segmenting pellets.

Morphological image processing is a filtering technique based on neighborhood operations. Such an operation analyses each point of a set within a specified neighbourhood of that point in order to produce a result. The elementary operations of mathematical morphology are two operations called erosion and dilation. Erosion is an neighbourhood operation that erodes the objects in the image based on the specified neighbourhood, and dilation is an operation that grows (dilates) the objects in the image based on the specified neighbourhood. These neighborhoods are defined by a user defined shape called a structuring element.

\subsection{Segmentation Method}

We apply the following process to identify all the individual pellets in the 3D surface data.

Collect Input Data: Sparse, and irregularly spaced $3 \mathrm{D}$ surface data of a stream of piled pellets on the conveyor belt.

Edge Detection 1: Find under-lapping edges and remove under-lapping data.

As a feature of the laser triangulation data capture system, some $(\mathrm{x}, \mathrm{y}, \mathrm{z})$ coordinate points are under-lapping along the $\mathrm{z}$ axis. This occurs at the center facing edge of some pellets that are typically located towards the edge of the conveyor belt. We use this underlapping information to find a few pellet edges and then we remove the under-lapping data as it is not suitable for the greyscale-based mathematical morphology (Thurley and $\mathrm{Ng}, 2005$ ) that we apply here.

Edge Detection 2: Edges of occluded areas

Sparse or occluded areas where there are no data points occur at the edges of pellets. By detecting discontinuities in the column pixel count from the camera we detect some edges of occluded areas.

Resample existing data sets to a regular grid.

After exploiting the raw data structure to remove under-lapping edges and identify occluded areas, we resample the data to a regular grid to improve computational efficiency in the subsequent processes.

Crop the width of the data to reduce bias due to increasing occlusion and diminishing data per pellet at the extremities of the laser line. 


\section{Edge Detection 3: Morphological laplacian}

To define the morphological laplacian we first provide the mathematical functional notation of erosion of an image $A$ by structuring element $B$ by $\epsilon_{B}(A)$ (Dougherty and Lotufo, 2003, pp. 5) and dilation by $\delta_{B}(A)$ (Dougherty and Lotufo, 2003, pp. 8).

We define the morphological laplacian as $\left(\delta_{B}(A)-\right.$ $A)-\left(A-\epsilon_{B}(A)\right)$ where the edges are defined by zero crossings. We incorporate this method as it performs well for detecting edges between overlapping pellets. We performed a morphological laplacian using a $0.8 \mathrm{~mm}$ radius spherical structuring element, took the absolute $\mathrm{z}$ value, performed a morphological closing (Dougherty and Lotufo, 2003, pp. 27) using a 0.8mm spherical closing to fill gaps, and then a $10 \%$ threshold on $\mathrm{z}$ depth.

\section{Edge Detection 4: Closing top hat}

The morphological closing top hat is a good general edge detector for particles. We performed the closing top hat (Dougherty and Lotufo, 2003, pp. 27) operation using a $1.6 \mathrm{~mm}$ radius spherical structuring element, followed by a $1.5 \%$ threshold on $\mathrm{z}$ depth.

\section{Edge Detection 5: Neighbourhood count}

We detect edges of sparse areas in the data by calculating the neighbour count for all data points using a $0.8 \mathrm{~mm}$ radius. Points with a low number of neighbours in the specified radius are likely edges of sparse areas in the data. We applied a threshold to retain only the smallest $40 \%$ of values from the neighbour count operation.

Combine Edges: Add all 5 edge detection methods together. Figure 2 shows a portion of the results of this operation.

\section{Distance Transform on the edge set.}

Using the combined edge set shown in figure 2 we calculate, for each $3 \mathrm{~d}$ point, the distance to the nearest edge point.

\section{Find Local Maxima in the Distance Transform}

We process the result of the distance transform and find local maxima using the morphological operation, opening by reconstruction (Dougherty and Lotufo, 2003 , pp. 136) with a $1.6 \mathrm{~mm}$ radius spherical structuring element for the opening, and a $0.8 \mathrm{~mm}$ radius flat circular disk for the reconstruction.

\section{Seed Growing from Local Maxima}

We use a seed growing (Thurley and $\mathrm{Ng}, 2005$ ) technique that expands the local maxima based on their height in the distance transform, merging spatially close maxima and creating seed regions for the watershed segmentation.

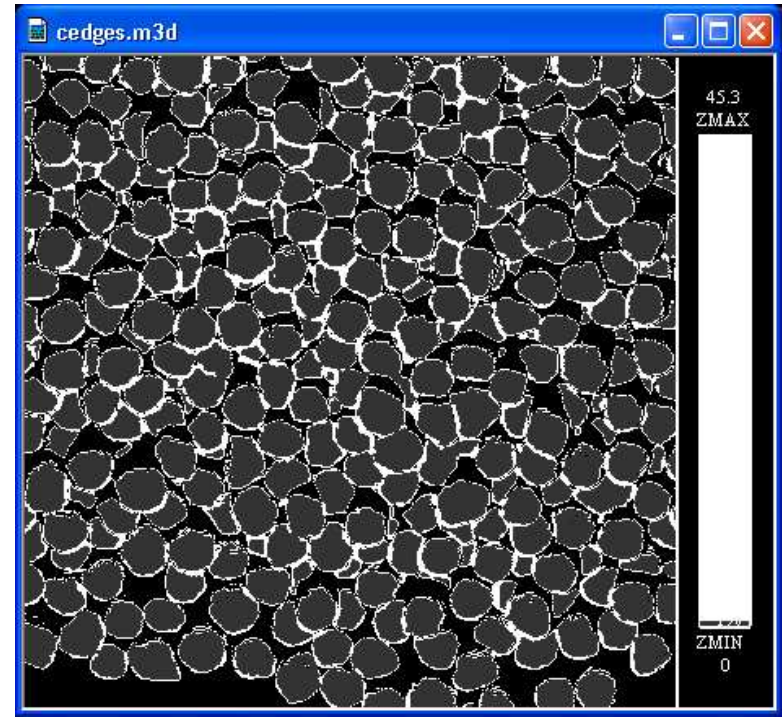

Fig. 2. A portion of the detected pellet edges combining 5 techniques

\section{Watershed Segmentation}

A seeded watershed (Dougherty and Lotufo, 2003, ch. 7) segmentation is performed to identify the individual pellet regions. This is a well established segmentation technique which is typically performed on the gradient representation of the input data. In this case we use the closing top hat edge set as the detected region boundaries are more accurate for this data than when the morphological gradient (Dougherty and Lotufo, 2003, pp. 48) edge set is used.

Post-filtering to remove tiny regions.

A sample segmentation is presented in figure 4 for the $3 \mathrm{D}$ data shown in figure 3 . The segmentation is of a pile of baked pellets collected in the laboratory as green pellets are prohibitively fragile for laboratory experimentation.

\section{Segmentation Results}

The segmentation identifies 1092 regions and after a region by region $3 \mathrm{D}$ visual analysis of the segmentation, $3 \mathrm{~d}$ data, and overlapping photograph of the pile, we provide the following qualitative assessment.

- Over-segmentation occurred in 5 pellets that were reported as 10 regions.

- Under-segmentation occurred in 34 pellets that were reported as 17 regions.

- A segmentation shift occurred in 4 pellets that were reported as 4 regions but the region was shifted sig- 


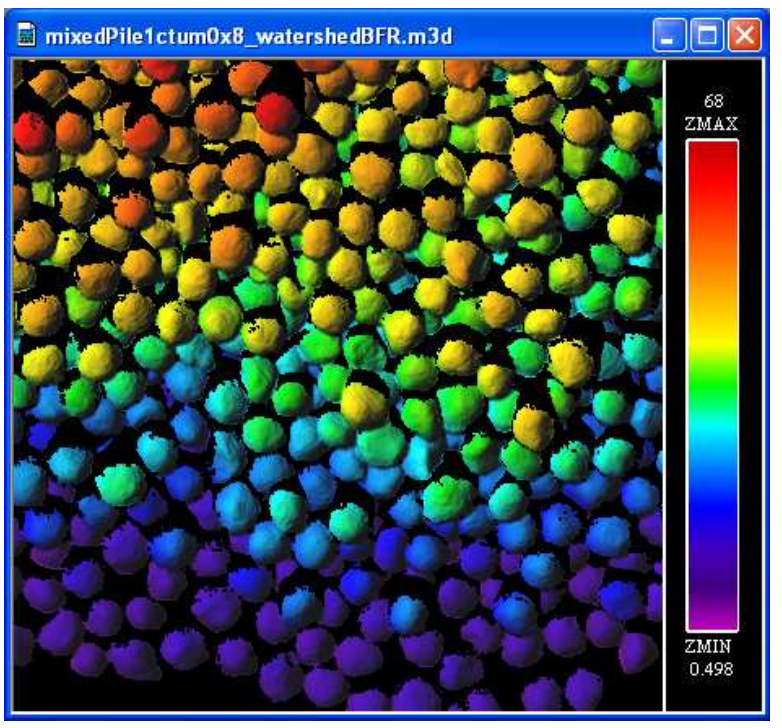

Fig. 3. 3D surface data of a portion of the pellet pile

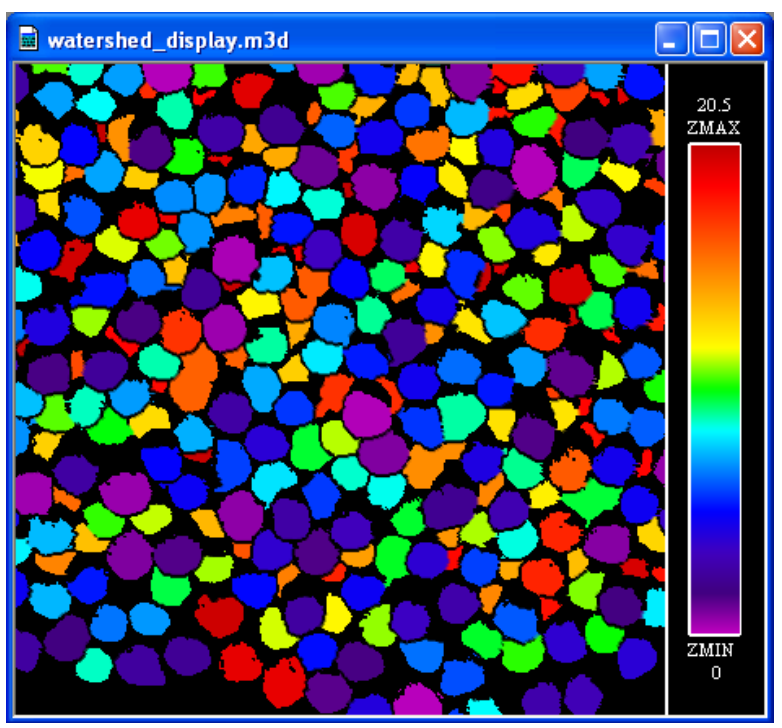

Fig. 4. Segmentation of a portion of the pellet pile

nificantly off the pellet. These regions corresponded to partially visible (overlapped) pellets.

- The 1092 detected regions represented 1104 pellets.

\section{Overcoming Overlapped Particle Error}

\subsection{Pellet Visibility}

Before we can accurately size the pellets detected by the segmentation we must make a determination of the pellets surface visibility for each region. That is, we must determine if a pellet is entirely visible, or it is overlapped by other pellets and is only partially visible.

This distinction between entirely and partially visible rock fragments has been performed for laboratory rock piles by Thurley (2002). This distinction is very powerful as it allows accurate size determination of entirely visible particles for which the entire profile is visible, and for partially visible particles to be ignored as their profile indicates little about the size of the entire rock, analogous to the tip of an iceberg. As a result we can overcome bias due to overlapped particle error.

By manually interrogating the 3D surface data of the laboratory pellet pile we define each region as either entirely or partially visible so as to define the "ground truth" for the purposes of creating an automatic classification algorithm.

We note that as pellets are consistently blob like in shape, we expect that 2D shape factors will offer additional features for classification of pellet visibility. Entirely visible pellets will typically appear approximately circular in shape when projected into the $x-y$ plane, whereas partially visible pellets should have regions with odd, convex, crescent-like and/or angular 2D shapes.

For the developed industrial system we used a combination of visibility ratio (Thurley, 2002) and a 2D non-perpendicular aspect ratio for pellet visibility classification. The non-perpendicular aspect ratio is equal to the major axis divided by the minor axis where the major and minor axes do not have to be perpendicular. A subsequent more rigorous investigation of visibility classification using a range of features is presented by Andersson et al. (2007b).

Using box-plots (Wikipedia, 2007b; Tukey, 1977) we depict the spread of the visibility ratio and aspect ratio distributions and consider their capacity for discriminating between entirely and partially visible pellets (figures 5 and 6).

A boxplot is a useful way of depicting a population of data without any assumptions of the statistical distribution. The central portion of a box-plot contains a rectangular box. In the center of this box is a short thick vertical black line which marks the median value (or 50 percentile) of the data. The left edge of the rectangular box marks the 25 percentile, and the right edge of the box marks the 75 percentile. The circular dots to the left and right of each box-plot indicate values that are statistically determined to be outliers. The graphs and determination of outliers were calculated using the $\mathrm{R}$ statistical analysis package (R Project, 2005). 
Region Visibility Ratio by Manual Visibility Classification

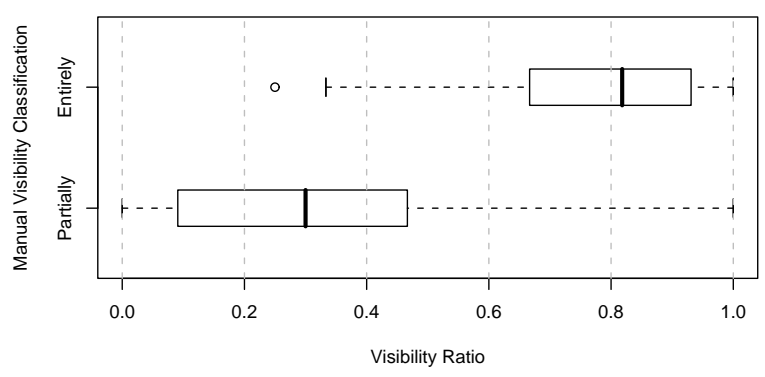

Fig. 5. Visibility ratio as a feature for discriminating between entirely and partially visible pellets.

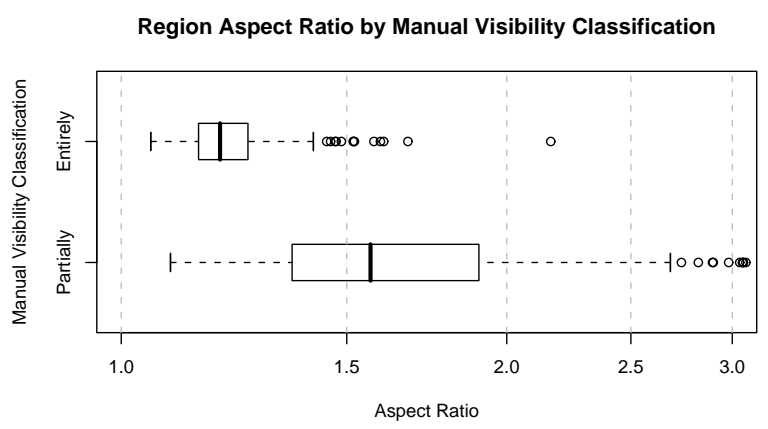

Fig. 6. Aspect ratio (non-perpendicular) as a feature for discriminating between entirely and partially visible pellets.

\subsection{Visibility Classification}

Both the visibility ratio (figure 5 ) and aspect ratio (figure 6) demonstrate a clear separation in the central box portion of the distributions for the entirely and partially visible pellets which suggests they are promising features for classification. We now plot both of these features in a 2D scatter plot (figure 7) and define a linear discriminant function to separate partially and entirely visible pellets.

The fisher linear discriminant (Duda et al., 2001, pp. 117) is one method for determining a linear discriminant function between two classes. We can use Fisher linear discriminant if "one is willing to sacrifice some of the theoretically attainable performance for the advantages of working in one dimension" (Duda et al., 2001, pp. 120). It projects a high dimension problem onto a line, reducing it to one dimension. In a two class problem, the projection maximises the distance between the mean of the two classes, while minimising the class variances. The resultant classifi- cation is a matter of selecting a threshold between the two classes in the one dimensional projection space. The resultant linear discriminant, is the line perpendicular to the one dimensional projection intersecting this threshold. Furthermore, we apply a weighted cost function to the determination of the threshold such that misclassification of partially visible pellets is 3 times more costly than misclassification of entirely visible pellets. We apply this weighted cost as it has little impact on our results to misclassify some entirely visible pellets for the benefit of preventing partially visible pellets being mistakenly classified and sized as entirely visible pellets. The resultant linear discriminant has a gradient of 0.4286 , a vertical axis intercept of 0.1844 and is shown figure 7 .

You will note in figure 7 that very few points corresponding to partially visible pellets are placed above the linear discriminant. When we come to sizing pellets, we will size only those pellets above the discriminant line, as we can be highly confident that regions above this line will correspond to entirely visible pellets for which we can confidently estimate size without bias due to overlapped particle error.

Region Visibility vs Aspect Ratio for Manual Visibility classifications

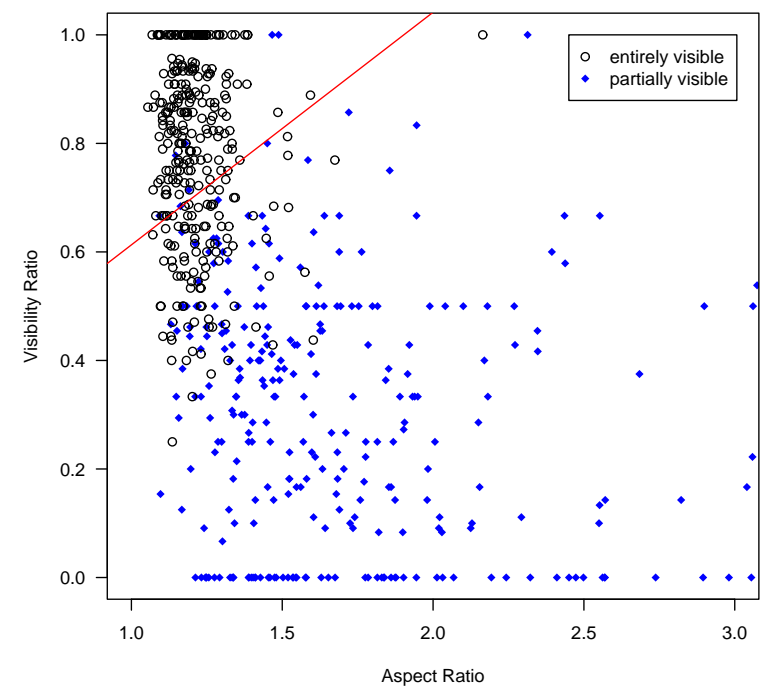

Fig. 7. Aspect ratio (non-perpendicular) vs Visibility ratio as a $2 \mathrm{D}$ feature space for discriminating between entirely and partially visible pellets.

\subsection{Visibility Validation}

Three validation techniques are typically considered when estimating the performance of classifiers; 
- Re-substitution method is a simple method where a classifier is tested on the same data upon which it was developed. This gives better results than if the classifier was tested on new data and provides an overestimate (sometimes an extreme overestimate) of the performance of the classifier.

- Holdout method is a method where a set of data is held out when developing the classifier. The classifier is developed on a separate set and may be tested on a new set of data to produce an unbiased estimate of classifiers performance. This is the most robust method but it is also requires the most data.

- Cross-validation is especially useful for providing a "nearly unbiased estimate of the true probabilities of correct and incorrect classifications" (Johnson, 1998, pg. 221 pa. 2) when the data available is limited.

As we did not have a second data set, we used 10fold cross-validation to get an estimate of how well the the visibility and sizing classification works. The method is an iterative process of developing a classifier and estimating the classifier's performance. At each iteration one part of the data set is held out to be a test set and the rest of the data is used to train the classifier. A 10-fold cross-validation performs the train-and-test iteration process 10 times. In each iteration, $10 \%$ of the data is is held out to be a testing set. An average of the classifier accuracy is then calculated for the 10 different classification results.

As shown in table 1 the expected performance of the classification strategy ensures that $98.57 \%$ of partially visible pellets are excluded, and $69.04 \%$ of entirely visible pellets are included. Using this classification strategy we will identify the entirely visible pellets that we will size in the absence in overlapped particle error.

\begin{tabular}{ccc}
\hline $\begin{array}{c}\text { "Ground truth" } \\
\text { classification }\end{array}$ & \multicolumn{2}{c}{ Predicted classification } \\
Entirely vis. & Partially vis. \\
\hline Entirely visible & 69.04 & 30.96 \\
Partially visible & 1.43 & 98.57 \\
\hline
\end{tabular}

Table 1

Confusion matrix generated with 10-fold cross validation for classification with a fisher linear discriminant based on a weighted cost function

\section{$3.4 \quad$ Sizing}

We define region size by using a measure of area calculated from the encompassing best-fit-rectangle (Wang et al., 1996; Wang, 2006) of a region. The bestfit-rectangle is calculated in the $\mathrm{x}-\mathrm{y}$ plane projection of each region and is the rectangle of minimum area that can completely encompass the region. We show the distributions of best-fit-rectangle area by size class for the surface pellets in our laboratory pile in figure 8 . We note that best-fit-rectangle provides a reasonable separation in the central portion of each boxplot with the significant exception being the close size classes $9 \mathrm{~mm}$ and $10 \mathrm{~mm}$ which show a stronger overlap. In all cases, the median value is clearly separable and the final results will demonstrate that best-fit-rectangle is a sufficient sizing feature. Further research work into complimentary size measurement features is ongoing.

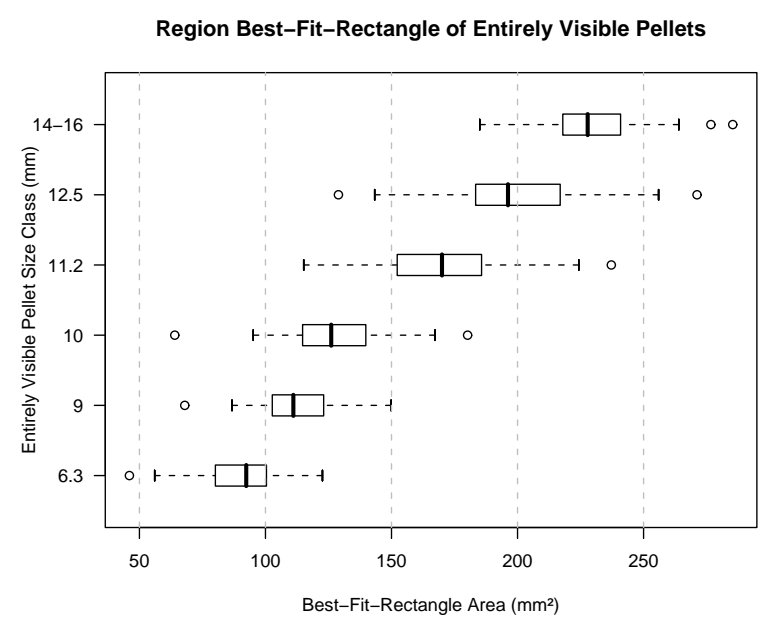

Fig. 8. Distributions of best-fit-rectangle area by size class for the "ground truth" manually defined entirely visible pellets.

\section{Industrial System}

\subsection{Training}

Using the described segmentation, visibility classification, and sizing methods developed on the laboratory pellet pile we process data from the industrial system and determine the best-fit-rectangle area for the pellets we consider to be entirely visible. What remains in order to produce a pellet size distribution is to determine a series of decision boundaries that classify best-fitrectangle area into the various sieve size classes.

To obtain the necessary data our industry partner collected a frequent and high resolution sieving, obtaining 23 samples over a $5 \frac{1}{2}$ hour period, of a portion of the pellet stream feeding onto the conveyor belt (table 2).

The sieving results used 10 size classes with lower bounds at $0,5,8,9,10,11,12.5,13,14$ and $16+\mathrm{mm}$. The material collected at size class $0-5 \mathrm{~mm}$ comprised 


\begin{tabular}{ccccccccccc}
\hline Time & & \multicolumn{7}{c}{ Sieve Size Class Lower Bound (mm) } \\
& 0 & 5 & 8 & 9 & 10 & 11 & 12.5 & 13 & 14 & $16+$ \\
\hline $\mathbf{9 : 0 1}$ & 0.51 & 0.63 & 0.74 & 3.93 & 24.75 & 45.06 & 12.24 & 8.31 & 3.60 & 0.24 \\
$\mathbf{9 : 1 0}$ & 0.00 & 0.55 & 1.53 & 6.66 & 39.52 & 30.81 & 11.07 & 7.67 & 2.18 & 0.00 \\
$\mathbf{9 : 2 5}$ & 0.21 & 0.32 & 0.74 & 3.81 & 26.02 & 40.68 & 10.57 & 13.50 & 3.79 & 0.36 \\
$\mathbf{9 : 3 5}$ & 0.45 & 0.90 & 1.88 & 10.60 & 36.21 & 36.56 & 5.28 & 4.94 & 2.75 & 0.43 \\
$\mathbf{9 : 4 5}$ & 0.55 & 0.84 & 0.79 & 5.21 & 34.04 & 39.75 & 8.35 & 7.57 & 2.58 & 0.32 \\
$\mathbf{9 : 5 5}$ & 0.41 & 0.99 & 1.61 & 5.94 & 34.86 & 37.85 & 7.50 & 6.58 & 4.27 & 0.00 \\
$\mathbf{1 1 : 2 4}$ & 0.29 & 0.42 & 1.38 & 4.86 & 29.51 & 40.68 & 8.73 & 10.86 & 3.27 & 0.00 \\
$\mathbf{1 1 : 3 4}$ & 1.10 & 1.71 & 2.08 & 9.44 & 34.61 & 36.90 & 5.34 & 6.00 & 2.83 & 0.00 \\
$\mathbf{1 1 : 4 4}$ & 0.58 & 1.77 & 3.62 & 12.11 & 40.54 & 29.92 & 7.21 & 3.58 & 0.68 & 0.00 \\
$\mathbf{1 1 : 5 5}$ & 0.34 & 0.73 & 2.17 & 8.66 & 39.32 & 34.35 & 6.69 & 5.62 & 2.12 & 0.00 \\
$\mathbf{1 2 : 0 5}$ & 0.51 & 0.97 & 1.18 & 7.76 & 34.88 & 35.95 & 9.12 & 8.57 & 1.06 & 0.00 \\
$\mathbf{1 2 : 1 5}$ & 0.47 & 0.47 & 0.94 & 3.89 & 32.61 & 41.12 & 10.65 & 6.65 & 2.92 & 0.29 \\
$\mathbf{1 2 : 3 4}$ & 0.38 & 0.67 & 0.78 & 3.61 & 25.80 & 34.85 & 15.95 & 13.59 & 3.57 & 0.79 \\
$\mathbf{1 2 : 5 1}$ & 0.53 & 0.92 & 0.71 & 3.77 & 25.83 & 35.05 & 12.40 & 13.21 & 6.20 & 1.38 \\
$\mathbf{1 3 : 0 1}$ & 0.58 & 0.42 & 0.11 & 2.10 & 16.85 & 44.25 & 13.64 & 15.19 & 6.11 & 0.76 \\
$\mathbf{1 3 : 1 1}$ & 0.29 & 0.33 & 0.33 & 2.00 & 21.65 & 43.07 & 16.35 & 11.92 & 2.70 & 1.36 \\
$\mathbf{1 3 : 2 1}$ & 0.67 & 0.55 & 0.33 & 1.59 & 16.39 & 41.06 & 15.92 & 14.93 & 7.93 & 0.65 \\
$\mathbf{1 3 : 3 1}$ & 0.48 & 0.42 & 0.19 & 1.49 & 16.81 & 34.75 & 19.98 & 18.77 & 5.94 & 1.17 \\
$\mathbf{1 3 : 4 3}$ & 0.80 & 1.45 & 3.19 & 9.87 & 38.28 & 32.34 & 7.41 & 5.36 & 1.31 & 0.00 \\
$\mathbf{1 3 : 5 3}$ & 0.33 & 0.24 & 0.91 & 6.60 & 33.08 & 40.93 & 10.92 & 4.98 & 1.99 & 0.00 \\
$\mathbf{1 4 : 0 3}$ & 0.95 & 0.73 & 0.75 & 4.70 & 30.29 & 40.19 & 12.39 & 9.29 & 0.71 & 0.00 \\
$\mathbf{1 4 : 1 3}$ & 0.69 & 0.61 & 1.38 & 6.93 & 27.98 & 42.98 & 8.96 & 7.51 & 2.97 & 0.00 \\
$\mathbf{1 4 : 2 3}$ & 0.36 & 0.93 & 1.41 & 5.54 & 29.98 & 39.51 & 10.33 & 9.07 & 2.86 & 0.00 \\
\hline & & & & & & & & &
\end{tabular}

Table 2

Sieving results ( $\%$ by weight) of iron ore green pellets during production

only of small fragments of broken pellets and is not significant for the pellet size measurement system. Therefore we leave this size class out of the final results. This is convenient for the vision system as one of the limitations of vision systems is the lower limit on resolution. In this case we have data points at approximately a $0.5 \mathrm{~mm}$ spacing and a smallest sieve size at $5 \mathrm{~mm}$ is at a size where we are completely confident we can detect pellets.

The measurements in table 2 are percentage by weight and it is necessary for us to convert this distribution to percentage by number to correspond with the pellet counts from our analysis. Once we have trained our decision boundaries on the percentage by number sieve distribution, we will convert our analysis result back to percentage by weight for the final presentation of results. In order to convert to percentage by number our industry partner measured some average weights per pellet using some of the sieving samples. We use weights for green pellets by size class as shown in table 3.

Once we have converted the sieve size class distributions to percentage by number we train our decision boundaries to these size classes. For a single sieving result it is a simple counting process to select decision boundaries that convert the distribution of best- fit-rectangle area values at that time into a near perfect result with the correct number of pellets in each size class. Calculating these decision boundaries for each of the first 6 sets of sieving data produces the results shown in table 4 . The purpose of presenting these decision boundaries is to show that decision boundaries that produce near perfect results at one time interval, are not necessarily similar as at another time interval.

In order to produce generic decision boundaries we need a method that can select the boundaries such that the resultant error is minimised. To achieve this we use the Nelder and Mead (1965) iterative error minimisation process. This optimisation method is available in the popular numerical computing package Matlab using function fminsearch, or in the statistical package $\mathrm{R}$ (R Project, 2005) using function optim which we use here. Given an initial estimate of the decision boundaries we use Nelder-Mead minimisation to produce a final set of boundaries that has minimum error. We note however, that the Nelder-Mead algorithm is sensitive to the initial estimate and can lead to inappropriate termination at non-optimal points" (Tomick et al., 1995). However, given a sufficient initial estimate we achieve suitable decision boundaries.

The Nelder-Mead optimisation takes an estimate of the decision boundaries and calculates the error at 
Table 3

\begin{tabular}{cccccccccc}
\hline \multicolumn{10}{c}{ Sieve Size Class Lower Bound $(\mathrm{mm})$} \\
0 & 5 & 8 & 9 & 10 & 11 & 12.5 & 13 & 14 & $16+$ \\
\hline 0.26 & 0.71 & 1.7 & 2.35 & 2.98 & 3.83 & 5.19 & 6.35 & 7.33 & 9.5 \\
\hline
\end{tabular}

Average weight $(\mathrm{g})$ for iron ore green pellets

\begin{tabular}{cccccccccc}
\hline Time & \multicolumn{8}{c}{ Sieve Size Class Lower Bound $(\mathrm{mm})$} \\
& 5 & 8 & 9 & 10 & 11 & 12.5 & 13 & 14 & $16+$ \\
\hline $\mathbf{9 : 0 1}$ & 74.00 & 79.13 & 80.75 & 87.50 & 109.92 & 146.98 & 167.90 & 195.64 & 278.72 \\
$\mathbf{9 : 1 0}$ & 15.75 & 59.50 & 67.83 & 83.82 & 117.76 & 146.32 & 165.07 & 195.16 & - \\
$\mathbf{9 : 2 5}$ & 59.38 & 69.07 & 72.00 & 82.31 & 108.66 & 143.00 & 156.00 & 190.56 & 248.69 \\
$\mathbf{9 : 3 5}$ & 56.13 & 68.00 & 72.25 & 86.90 & 115.00 & 154.34 & 168.25 & 188.47 & 224.86 \\
$\mathbf{9 : 4 5}$ & 70.44 & 76.50 & 78.39 & 85.53 & 110.35 & 148.55 & 162.00 & 185.02 & 239.30 \\
$\mathbf{9 : 5 5}$ & 59.89 & 71.03 & 75.87 & 83.87 & 112.62 & 147.73 & 160.99 & 181.69 & - \\
\hline Median & 59.64 & 70.05 & 74.06 & 84.70 & 111.48 & 147.36 & 163.54 & 189.52 & 244.00 \\
Nelder & 60.17 & 68.66 & 74.03 & 84.55 & 111.91 & 147.94 & 164.73 & 197.17 & 255.16 \\
\hline
\end{tabular}

Table 4

Decision boundaries $\left(\mathrm{mm}^{2}\right)$ for best-fit-rectangle that generate near-perfect sieving results at each time interval, the Median values, and the optimised result from Nelder-Mead minimisation

that estimate. It then modifies the decision boundaries slightly following a range of internal rules before repeating the process. Changes that reduce the error are kept, while changes that increase the error are typically discarded. We use the median of the decision boundaries in table 4 as the initial estimates for the Nelder-Mead and generate the optimised result, also shown at the bottom of table 4 .

One of the complications with the error minimisation is that it is being performed on the percentage by number distributions, but what we actually want to minimise is the error in the final results, which will be percentage by weight distributions. As a result we scale the error calculations during the Nelder-Mead minimisation by the size class weights from table 3 . We express the error function as a sum of squares as shown in equation 1 .

$\epsilon=\sum_{t}\left[\sum_{i=1}^{N}\left[\left(C_{i, t}-S_{i, t}\right) W_{i}\right]^{2}\right]$

$\begin{array}{ll}t & \text { Time of day } \\ i & \text { Sieve size class } \\ N & \text { Number of sieve size classes } \\ C_{i, t} & \text { Calculated value of the size class } i \text { at time } t \\ S_{i, t} & \text { Sieving result for size class } i \text { at time } t \\ W_{i} & \text { Average pellet weight of size class } i\end{array}$

Using the optimised Nelder-Mead decision boundaries from table 4 we produce estimates of the sieve size distributions across the $5 \frac{1}{2}$ hour period for which we have measured sieve data. The results are presented in figure 9. The left block of the graph is shaded gray to show the portion of the sieving data that was used to train the decision boundaries, with the remaining 17 sieve results illustrating the capacity of the system to estimate the sieve size.

The results show a strong capacity to estimate sieve size (with some error) even at the presented high resolution sieve analysis where 5 size classes have a spacing of only $1 \mathrm{~mm}$ and the $12.5-13 \mathrm{~mm}$ size class only a $0.5 \mathrm{~mm}$ spacing. The imaging results are calculated for every minute between 9:00 and 14:30 and total 331 calculations. The plots of the imaging results are running averages across a 3 minute interval.

We note also that the image processing results show interesting variation between each sieving measurement across the day which is one of the advantages of a continuous online measurement system. In the future this will allow for a previously unattainable high resolution examination of pellet size variation during production and lead to a much better understanding and control of the pellet balling disk/drum.

\section{Future research}

The presented research provides fast feedback to the pelletising process with high resolution sieving results. This opens the door to autonomous closed loop control of the pellet balling disk/drum and we expect advances in this area in the near future.

Application of the presented system to measurement of baked pellets prior to charging the blast furnace in steel production is an immediately applicable application. Some additional research may be necessary to investigate the varying conditions produced by baked 


\section{Iron Ore Green Pellet Size Distribution}
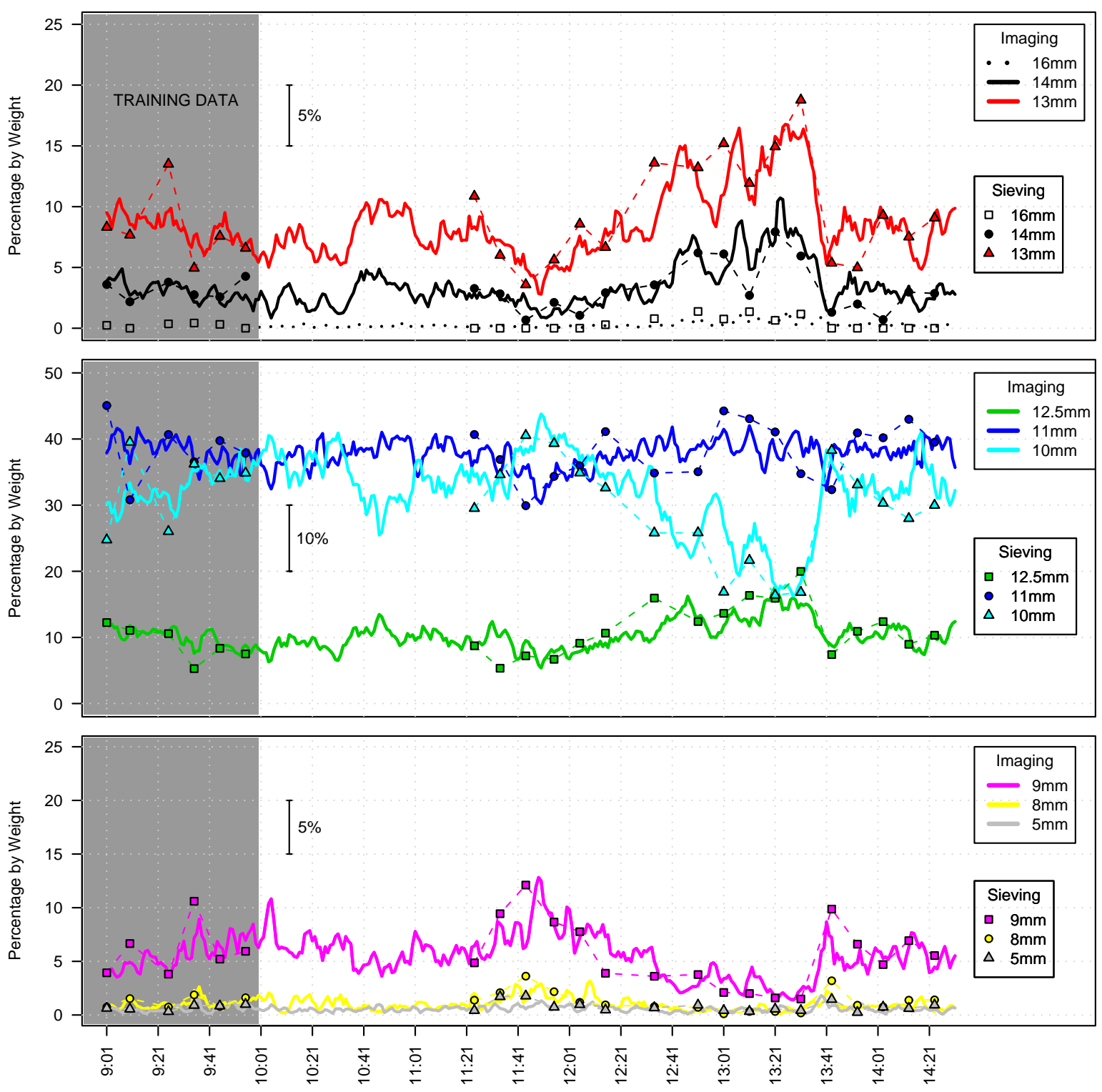

Fig. 9. Imaging and sieving results for $5 \frac{1}{2}$ hours of production where the speed of the pelletising disk was being changed.

pellets specifically due to segregation error / brazil nut effect (Rosato et al., 1987; Wikipedia, 2007c).

Extending the presented system to rocks on conveyor belt is also planned based on the existing research of Thurley (2002) on laboratory rock piles.

Further research into features for size measurement and classification is also of interest, specifically to establish sizing features that can complement best-fitrectangle (Wang, 2006).

\section{Comments}

The high speed camera ensures that we have a high density of $3 \mathrm{D}$ points at approximately a spacing of $0.5 \mathrm{~mm}$ in the plane of the conveyor belt. Having a data density at this high resolution has at least two advantages. Firstly it allows us to detect small sliver or crescent like regions of overlapped particles and ensure that they are not merged into other regions. 
And secondly it ensured that we had a high resolution when it came to measuring the size of each pellet, and allowed us to produce a size distribution with very fine spacing, specifically, 5, 8, 9, 10, 11, 12.5, 13, 14 and $16 \mathrm{~mm}$ size classes. Notably there are 4 size classes that are only $1 \mathrm{~mm}$ wide, and one size class (12.5 $13 \mathrm{~mm}$ ) that is only $0.5 \mathrm{~mm}$ wide. Without the high speed camera generating this high resolution data it is the authors opinion that we could not have identified these size classes so effectively.

Furthermore, we can say that the system achieves what a number of commercial 2D fragmentation measurement systems could not satisfactorily achieve for the pellet producer, that is, accurate sizing of the green pellets.

Equivalent vision hardware can now be purchased offthe-shelf for about 15,000 Euros, and the current system has been running for 14 months without the need for maintenance.

The product is now in a commercialisation phase. Commercial enquiries can be directed to the primary author matthew. thurley@ltu. se and these will be forwarded on to our commercial partner.

\section{Conclusion}

An industrial prototype 3D imaging system for sizing of iron ore green pellets on conveyor belt has been developed with the pellet sieve size distribution determined into 9 sieve size classes between $5 \mathrm{~mm}$ and $16+\mathrm{mm}$. The advantages for the pellet production plant are fast, frequent, non-contact, consistent, high resolution measurement, and the potential for closed loop control of the pellet balling disk/drum. Pellet identification in the 3D surface data has been performed using morphological segmentation and a new two feature classification applied to identify entirely visible and partially visible (overlapped) pellets. Visibility classification is an important distinction of this work as it allows entirely visible pellets to be sized accurately, partially visible (overlapped) pellets to be ignored, and prevents bias due to the sizing of partially visible pellets based on their limited visible profile. Literature review indicates that no other researchers in particle size measurement for pellets or rocks make this distinction between entirely and partially visible particles. Sizing of the entirely visible pellets is performed using best-fit-rectangle which is classified into 9 sieve size classes from $5 \mathrm{~mm}$ up to and including $16+\mathrm{mm}$. This classification is trained on approximately one quarter of the sieving data using Nelder and Mead (1965) error minimisation with the resulting graphs of pellet size class illustrating a strong correlation between the imaging results and the sieving samples.

\section{Acknowledgments}

Thanks go to the Swedish research funding body VINNOVA, our industry partners, LKAB, Boliden Mining, and SSAB Steel, and to the staff at ProcessIT Innovations for all their efforts in arranging and facilitating the 3D measurement project. Thanks also to John Erik Larsson at MBV-systems for his efforts on the 3D surface imaging system and in particular our demands for additional hardware capabilities in the laboratory system, and to Kjell-Ove Mickelsson at LKAB and Robert Johansson at SSAB for their generous provisions of time, energy and invaluable industry advice.

\section{References}

Andersson, T., Thurley, M., Marklund, O., May 2007a. Pellet size estimation using spherical fitting. In: Proceedings of the IEEE Instrumentation and Measurement Technology Conference. IEEE, pp. 1-5.

Andersson, T., Thurley, M., Marklund, O., December 2007b. Visibility classification of pellets in piles for sizing without overlapped particle error. In: Digital Image Computing: Techniques and Applications Conference Proceedings, DICTA 2007. Australian Pattern Recognition Society, accepted.

Blomquist, M., Wernerson, A., September 1999. Range camera on conveyor belts: Estimating the size distribution and systematic errors due to occlusion. In: Proceedings of the SPIE - The International Society for Optical Engineering. pp. 118-126.

Bouajila, A., Bourassa, M., Boivin, J., Ouellet, G., Martinovic, T., 1999. On-line non-intrusive measurement of green pellet diameter. In: Ironmaking Conference Proceedings. pp. 10091020 .

Chavez, R., Cheimanoff, N., Schleifer, J., August 1996. Sampling problems during grain size distribution measurements. In: Proceedings of the Fifth International Symposium on Rock Fragmentation by Blasting - FRAGBLAST 5. Montreal, Quebec, Canada, pp. 245-252

Dougherty, E. R., Lotufo, R. A., 2003. Hands-On Morphological Image Processing. Vol. TT59. SPIE - The International Society for Optical Engineering.

Duda, R. O., Hart, P. E., Stork, D. G., 2001. Pattern Classification, 2nd Edition. Wiley, ISBN 0-471-05669-3.

Explo 2001, October 2001. Explosives in Mining Conference. AusIMM, Hunter Valley, NSW, Australia.

Franklin, J., Katsabanis, T. (Eds.), 1996. Measurement of Blast Fragmentation - Proceedings of the FRAGBLAST 5 Workshop. Balkema.

Frydendal, I., Jones, R., August 1998. Segmentation of sugar beets using image and graph processing. In: ICPR 98 Proceedings $14^{t h}$ International Conference on Pattern Recognition. Vol. II. Brisbane, Australia, pp. 16-20.

Johnson, D. E., 1998. Applied Multivariate Methods for Data Analysts. Duxbury Press, ISBN 0-534-23796-7.

Kemeny, J., Mofya, E., Kaunda, R., Lever, P., October 2001. Improvements in blast fragmentation models using digital image processing. In: Explo 2001 (2001), pp. 357-363.

Kim, H., Haas, C., Rauch, A., Browne, C., 2003. 3d image segmentation of aggregates from laser profiling. Computer Aided Civil and Infrastructure Engineering, 254-263.

Lee, J., Smith, M., Smith, L., Midha, P., 2005. A mathematical 
morphology approach to image based $3 \mathrm{~d}$ particle shape analysis. In: Machine Vision and Applications. Vol. 16(5). SpringerVerlag, pp. 282-288.

Maerz, N., Palangio, T., Franklin, J., 1996. Wipfrag image based granulometry system. In: Franklin and Katsabanis (1996), pp. 91-99.

Nelder, J., Mead, R., 1965. A simplex method for function minimisation. The Computer Journal 7, 308-313.

Ökvist, L., Dahlstedt, A., Hallin, M., 2001. The effect on blast furnace process of changed pellet size as a result of segregation in raw material handling. In: Ironmaking Conference Proceedings. pp. 167-178.

R Project, 2005. R: A language and environment for statistical computing. R Foundation for Statistical Computing, Vienna, Austria, ISBN 3-900051-07-0.

URL http://www.R-project.org

Rosato, A., Strandburg, K., Prinz, F., Swendsen, R., 1987. Why the brazil nuts are on top: Size segregation of particulate matter by shaking. Physical Review Letters 58 (10).

Salinas, R. A., Raff, U., Farfan, C., February 2005. Automated estimation of rock fragment distributions using computer vision and its applcation in mining. In: IEE Proceedings: Vision, Image and Signal Processing. Vol. 152(1). pp. 1-8.

Schleifer, J., Tessier, B., October 2001. Fragmentation assessment using the Fragscan system: Quality of a blast. In: Explo 2001 (2001), pp. 365-372.

Thurley, M., Ng, K., February 2005. Identifying, visualizing, and comparing regions in irregularly spaced $3 \mathrm{~d}$ surface data. Computer Vision and Image Understanding 98 (2), 239-270.

Thurley, M., Ng, K., Minack, J., December 1999. Mathematical morphology implemented for discrete, irregular 3d surface data. In: DICTA 99, Digital Image Computing: Techniques and Applications Conference Proceedings. Fifth Biennial Conference. Australian Pattern Recognition Society, Curtin University, Perth, Western Australia, pp. 16-20.

Thurley, M. J., December 2002. Three dimensional data analysis for the separation and sizing of rock piles in mining. Ph.D. thesis, Monash University.

URL http://image3d6.eng.monash.edu.au/thesis.html

Tomick, J., Arnold, S., Barton, R., 1995. Sample size selection for improved nelder-mead performance. In: Proceedings of the Winter Simulation Conference. pp. 341-345.

Tukey, J. W., 1977. Exploratory Data Analysis. Addison-Wesley, Ch. "Box-and-Whisker Plots" 2C, pp. 39-43, ISBN 0-20107616-0

Wang, W., 2006. Image analysis of particles by modified ferret method - best-fit rectangle. Power Technology, 1-10.

Wang, W., Bergholm, F., Stephansson, O., 1996. Image analysis of fragment size and shape. In: Franklin and Katsabanis (1996), pp. 233-243.

Wikipedia, July 2007a. 3d scanner. URL http://en.wikipedia.org/wiki/3d_scanner

Wikipedia, March 2007b. Boxplot. URL http://en.wikipedia.org/wiki/Boxplot

Wikipedia, June 2007c. Brazil nut effect.

URL http://en.wikipedia.org/wiki/Brazil_nut_effect 